\title{
A $K$-theoretic proof of Boutet de Monvel's index theorem for boundary value problems
}

\author{
By Severino T. Melo at São Paulo, Thomas Schick at Göttingen \\ and Elmar Schrohe at Hannover
}

\begin{abstract}
We study the $C^{*}$-closure $\mathfrak{A}$ of the algebra of all operators of order and class zero in Boutet de Monvel's calculus on a compact connected manifold $X$ with boundary $\partial X \neq \emptyset$. We find short exact sequences in $K$-theory

$$
0 \rightarrow K_{i}(C(X)) \rightarrow K_{i}(\mathfrak{A} / \mathfrak{A}) \stackrel{p}{\rightarrow} K_{1-i}\left(C_{0}\left(T^{*} X^{\circ}\right)\right) \rightarrow 0, \quad i=0,1
$$

which split, so that $K_{i}(\mathfrak{U} / \mathfrak{\Re}) \cong K_{i}(C(X)) \oplus K_{1-i}\left(C_{0}\left(T^{*} X^{\circ}\right)\right)$. Using only simple $K$ theoretic arguments and the Atiyah-Singer index theorem, we show that the Fredholm index of an elliptic element in $\mathscr{A}$ is given by

$$
\text { ind } A=\operatorname{ind}_{t}(p([A])) \text {, }
$$
\end{abstract}

where $[A]$ is the class of $A$ in $K_{1}(\mathfrak{U} / \mathfrak{i})$ and $\operatorname{ind}_{t}$ is the topological index, a relation first established by Boutet de Monvel by different methods.

\section{Introduction}

Boutet de Monvel's calculus is a pseudodifferential calculus on manifolds with boundary. It comprises the classical differential boundary value problems as well as the parametrices to elliptic elements, even their inverses whenever they exist [2]. If the underlying manifold is compact, elliptic operators in the calculus define Fredholm operators between appropriate Hilbert spaces. Boutet de Monvel established an index theorem for that case: He showed that there is a map which associates to each elliptic operator an element in the $K$-theory of the cotangent bundle over the interior of the manifold and that the Fred-

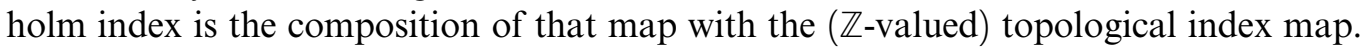

The crucial step is the construction of the above map from elliptic operators to $K$-theory, for which Boutet de Monvel uses elaborate considerations, combining homotopy arguments within the algebra with classical (vector-bundle) $K$-theory. In this article we will show that this map can be obtained relying only on basic knowledge of the structure of the 
algebra and relatively simple constructions in $K$-theory for $C^{*}$-algebras (which were not yet available in 1971). Boutet de Monvel's map is neither obvious nor trivial. The point is that we are able to represent it as a composition of various standard (yet non-trivial) maps in $K$-theory.

To be more specific, let $X$ be a compact $n$-dimensional manifold with boundary $\partial X$, embedded in a closed manifold $\tilde{X}$ of the same dimension ( $\tilde{X}$ could e.g. be the double of $X$ ). By $X^{\circ}$ we denote the interior of $X$. We assume that $X$ is connected and $\partial X$ is nonempty.

Given a pseudodifferential operator $P$ on $\tilde{X}$, we define the truncated pseudodifferential operator $P_{+}: C^{\infty}(X) \rightarrow C^{\infty}\left(X^{\circ}\right)$ as the composition $r^{+} P e^{+}$, where $e^{+}$is extension by zero from $X$ to $\tilde{X}$ and $r^{+}$is the restriction to $X^{\circ}$. In general, the functions in the range of $P_{+}$will not be smooth up to the boundary. One therefore assumes that $P$ satisfies the transmission condition, a condition on the symbol of $P$ which ensures that both $P_{+}$and $\left(P^{*}\right)_{+}$, the truncated operator formed from the formal adjoint of $P$, map smooth functions on $X$ to smooth functions on $X$.

An operator in Boutet de Monvel's calculus is a matrix

$$
A=\left(\begin{array}{cc}
P_{+}+G & K \\
T & S
\end{array}\right): \begin{gathered}
C^{\infty}\left(X, E_{1}\right) \\
C^{\infty}\left(\partial X, F_{1}\right)
\end{gathered} \rightarrow \begin{gathered}
C^{\infty}\left(X, E_{2}\right) \\
C^{\infty}\left(\partial X, F_{2}\right)
\end{gathered}
$$

acting on sections of vector bundles $E_{1}, E_{2}$ over $X$ and $F_{1}, F_{2}$ over $\partial X$. Here, $P$ is a pseudodifferential operator satisfying the transmission condition; $G$ is a singular Green operator, $T$ is a trace operator, $K$ is a potential (or Poisson) operator, and $S$ is a pseudodifferential operator on $\partial X$. All these operators are assumed to be classical; i.e. their symbols have polyhomogeneous expansions in the respective classes. For details, the reader is referred to the monographs by Rempel and Schulze [8] or Grubb [4] as well as to the short introduction [11]. We will need the following facts:

The operators $G, K$, and $T$ are regularizing in the interior of $X$. In a collar neighborhood of the boundary, they can be viewed as operator-valued pseudodifferential operators along the boundary. In particular, they have an order assigned to them. The singular Green and the trace operators also have a class (or type) $d \in \mathbb{N}_{0}$, related to the order of the derivatives appearing in the boundary condition.

The composition of two operators of the form (1) is defined whenever the vector bundles serving as the range of the first operator form the domain of the second. The composition $A A^{\prime}$ of an operator $A^{\prime}$ of order $m^{\prime}$ and class $d^{\prime}$ with an operator $A$ of order $m$ and class $d$ results in an operator of order $m+m^{\prime}$ and class $\leqq \max \left(m^{\prime}+d, d^{\prime}\right)$. In particular, the composition of two operators of order and class zero is again of order and class zero.

For $E_{1}=E_{2}=E$ and $F_{1}=F_{2}=F$, the operators of order and class zero thus form an algebra $\mathscr{A}$. Moreover, they extend to bounded operators on the Hilbert space $\mathfrak{H}=L^{2}(X, E) \oplus L^{2}(\partial X, F)$. In fact, $\mathscr{A}$ is a $*$-subalgebra of $\mathfrak{L}(\mathfrak{H})$, closed under holomorphic functional calculus, cf. [10]. We use here the definition of order and class in [8] and [11] which differs slightly from that in [4]. It allows us to use the $L^{2}$-space over the bound- 
ary instead of $H^{-1 / 2}$ and gives us better homogeneity properties of the boundary symbols. In view of the fact that both the kernel and the cokernel of an elliptic operator in $\mathscr{A}$ consist of smooth functions, the choice is irrelevant for index theory.

Standard reductions - recalled in Section 1.1 - allow to reduce any index problem to the case where the operator is an element of the algebra $\mathscr{A}$, so that we can apply operatoralgebraic methods. This is a central point of the paper.

Let us have a closer look at the structure of $\mathscr{A}$. In a generalization of the classical Lopatinskij-Shapiro condition, the ellipticity of an element $A \in \mathscr{A}$ is governed by the invertibility of two symbols, namely the pseudodifferential principal symbol, $\sigma(A)$, and the principal boundary symbol, $\gamma(A)$, which take values in certain $C^{*}$-algebras over $S^{*} X$ and $S^{*} \partial X$, respectively, cf. Section 1.2 .

The maps $\sigma$ and $\gamma$ are $*$-homomorphisms on $\mathscr{A}$. Extending the classical results by Gohberg and Seeley, Rempel and Schulze ([8], 2.3.4.4, Theorem 1, based on work by Grubb and Geymonat [5]) showed that

$$
\inf _{C \in \mathfrak{S}}\|A+C\|=\max \{\|\sigma(A)\|,\|\gamma(A)\|\}, \quad \text { for all } A \in \mathscr{A},
$$

where $\mathfrak{S}$ denotes the ideal of the compact operators on $\mathfrak{H}$, and the norms on the right-hand side are the supremum norms on $S^{*} X$ and $S^{*} \partial X$, respectively.

We shall now denote by $\mathfrak{A}$ the closure (equivalently, the $C^{*}$-closure) of $\mathscr{A}$ in the topology of $\mathfrak{L}(\mathfrak{H})$. It follows from (2) that $\sigma$ and $\gamma$ have continuous extensions to $\mathfrak{A}$; we denote them by the same letters. An element of $\mathfrak{U}$ is compact, if and only if both symbols are zero. Moreover, it is a Fredholm operator if and only if it is elliptic, i.e., both symbols are (bundle) isomorphisms.

Boutet de Monvel showed that, given an elliptic element $A$ in $\mathscr{A}$, one can find a homotopy through elliptic elements in $\mathscr{A}$, connecting $A \oplus \mathrm{Id}$ to an operator of the form $\left(\begin{array}{cc}P_{+}^{\prime} & 0 \\ 0 & Q^{\prime}\end{array}\right)$, where $P^{\prime}$ is a pseudodifferential operator whose principal symbol is an isomorphism of $E$ (independent of $\xi$ ) in a neighborhood of the boundary. Through the usual difference bundle construction, the principal symbol of $P^{\prime}$ therefore defines an element $\left[P^{\prime}\right]$ of $K\left(T^{*} X^{\circ}\right)$. Boutet de Monvel then associated to $A$ the class $\left[P^{\prime}\right]+\operatorname{Thom}\left(\left[Q^{\prime}\right]\right)$, where $\left[Q^{\prime}\right]$ is the class in $K\left(T^{*} \partial X\right)$ defined by the principal symbol of $Q^{\prime}$ and Thom : $K\left(T^{*} \partial X\right) \rightarrow K\left(T^{*} X^{\circ}\right)$ is the Thom map, also called Gysin homomorphism, or Umkehrmap. Moreover, he showed that the composition of that map with the topological index map $K\left(T^{*} X^{\circ}\right) \rightarrow \mathbb{Z}$ gives the index of $A$.

Fedosov [3] then proved that this implies the formula

$$
\text { ind } A=\int_{T^{*} X} \operatorname{ch}(\sigma(A)) \mathscr{T}(X)+\int_{T^{*} \partial X} \operatorname{ch}^{\prime}(\gamma(A)) \mathscr{T}(X) .
$$

Here $\mathscr{T}(X)$ is the differential form given by the Todd class of the tangent bundle (or its restriction to the boundary), $\operatorname{ch} \sigma(A)$ is the Chern character associated with the $K$-class induced by the pseudodifferential principal symbol $\sigma(A)$, and $\operatorname{ch}^{\prime} \gamma(A)$ is a differential form 
constructed from the $K$-class of the boundary symbol; it is given by a formula analogous to that for the usual Chern character.

In order to establish this formula, Fedosov referred to Boutet de Monvel's work and showed two facts: (i) the formula is invariant under homotopies within the class of elliptic boundary value problems in the calculus and (ii) whenever the principal symbol of $P$ is an isomorphism in a neighborhood of the boundary in $X$, independent of the covariable $\xi$, and the boundary symbol is an isomorphism over the full ball bundle $B^{*}(\partial X)$, then the above formula reduces to the classical formula of Atiyah and Singer.

Our approach to the index theorem is based on a careful analysis of the boundary symbol map. Indeed, since the kernel of $\gamma$ contains the compact operators, we have a natural short exact sequence

$$
0 \rightarrow \operatorname{ker} \gamma / \mathfrak{A} \rightarrow \mathfrak{A} / \mathfrak{K} \stackrel{\gamma}{\rightarrow} \operatorname{im} \gamma \cong \mathfrak{U} / \operatorname{ker} \gamma \rightarrow 0 .
$$

What is mainly needed for the understanding of our proof of the index theorem is the structure of $\operatorname{ker} \gamma$ and $\operatorname{im} \gamma$. Both were determined in [7], Section 3 for the case of trivial onedimensional bundles $E$ and $F$. We shall review these computations in Section 1 for the case of general bundles.

In Section 2, we recall a basic construction from $K$-theory, namely how a commutative diagram of short exact sequences of $C^{*}$-algebras

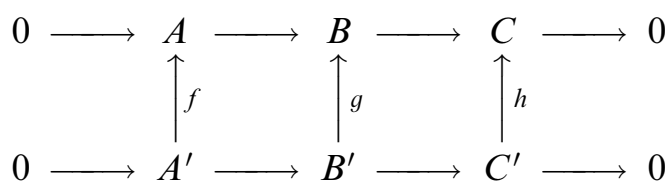

yields a commutative grid of $C^{*}$-algebras involving $A^{\prime}, B^{\prime}, C^{\prime}$, the mapping cones, and the suspensions of $A, B$, and $C$. We then apply this in Section 3 to the above sequence (4), linked to the sequence

$$
0 \rightarrow C_{0}\left(X^{\circ}\right) \rightarrow C(X) \rightarrow C(\partial X) \rightarrow 0
$$

As a first result, we obtain

Theorem 1. We have natural short exact sequences

$$
0 \rightarrow K_{i}(C(X)) \rightarrow K_{i}(\mathfrak{A} / \mathfrak{R}) \stackrel{p}{\rightarrow} K_{1-i}\left(C_{0}\left(T^{*} X^{\circ}\right)\right) \rightarrow 0
$$

$i=0,1$, which split; i.e. we have (not necessarily natural) isomorphisms

$$
K_{i}(\mathfrak{U} / \mathfrak{R}) \cong K_{i}(C(X)) \oplus K_{1-i}\left(C_{0}\left(T^{*} X^{\circ}\right)\right)
$$

This strengthens the results of Melo, Nest, and Schrohe [7], Corollaries 12 and 19, where the corresponding statements were derived for $i=0$ under an additional hypothesis and for $i=1$ using Boutet de Monvel's index theorem. 
In Section 4 we then prove the index theorem:

Theorem 2. The index of a Fredholm operator $A$ in $\mathfrak{A}$ is given by

$$
\text { ind } A=\operatorname{ind}_{t}(p([A])) \text {, }
$$

where $[A]$ is the $K_{1}$-class of $A$ in $\mathfrak{A} / \mathfrak{R}, p$ is the map in (5) and

$$
\operatorname{ind}_{t}: K_{0}\left(C_{0}\left(T^{*} X^{\circ}\right)\right) \cong K^{0}\left(T^{*} X^{\circ}\right) \rightarrow K^{0}(p t) \cong \mathbb{Z}
$$

is the topological index. Moreover, $p[A]=\operatorname{ind}(A)$, where ind denotes the map defined by Boutet de Monvel in [2], Theorem 5.21.

As in the classical case, Fedosov's arguments yield the cohomological form (3) of the index theorem.

\section{Elliptic operators and symbols in Boutet de Monvel's calculus}

1.1. A normal form for the index problem. Suppose we are given a Fredholm operator $A$ in Boutet de Monvel's calculus of order $m$ and class $d$ acting on sections of vector bundles over $X$ as in (1).

There exist order and class reducing invertible operators in the calculus. By invertibility, composition with those does not change the index. Therefore, we can always achieve that order and class are zero.

Moreover, we can always assume that $X$ is connected. If it is not, then the spaces $C^{\infty}\left(X, E_{1}\right), \ldots$, decompose as direct sums corresponding to the decomposition in connected components, and the operator $A$ becomes a matrix $M$ with respect to this decomposition. By definition of the Boutet de Monvel calculus, the off-diagonal entries are smoothing operators, thus compact. The index is therefore unchanged if we replace $M$ by the diagonal matrix equal to the diagonal of $M$. But then it is clear that the index is additive, and the individual entries correspond to the connected components of $X$.

We can also assume that $E_{1}=E_{2}=E$ and $F_{1}=F_{2}=F$. Indeed, if an elliptic Boutet de Monvel operator between $\left(E_{1}, F_{1}\right)$ and $\left(E_{2}, F_{2}\right)$ exists, we have in particular an interior elliptic pseudodifferential operator $Q$ between $E_{1}$ and $E_{2}$. By definition, its symbol defines an isomorphism between $\pi^{*} E_{1}$ and $\pi^{*} E_{2}$, where $\pi: S^{*} X \rightarrow X$ is the projection of the unit cotangent sphere bundle. Since $X$ has a non-empty boundary, $\pi: S^{*} X \rightarrow X$ has a section, see e.g. [7], Proposition 9, or use that the Euler class of the cotangent bundle is trivial. Restricting the symbol isomorphism to this section, we get a bundle isomorphism between $E_{1}$ and $E_{2}$.

Next we choose a complement $\tilde{E}$ to $E$ such that $E \oplus \tilde{E}=\underline{N}$, the $N$-dimensional trivial bundle. We can take $N$ so large that, over $\partial X$, the bundles $F_{1}$ and $F_{2}$ are also embedded in the restriction of $\underline{N}$ and consider the operator 


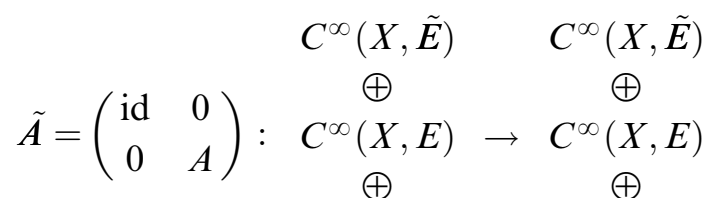

$$
\begin{aligned}
& C^{\infty}\left(\partial X, F_{1}\right) \quad C^{\infty}\left(\partial X, F_{2}\right)
\end{aligned}
$$

which has the same index as $A$.

We then recall that there are elliptic operators $R_{j}, j=1,2$, of order and class zero in Boutet de Monvel's calculus such that

$$
R_{j}=\left(\begin{array}{c}
\Gamma_{F_{j}} \\
p_{F_{j}} \circ \gamma_{0}
\end{array}\right): C^{\infty}(X, \underline{N}) \rightarrow \begin{gathered}
C^{\infty}(X, \underline{N}) \\
C^{\infty}\left(\partial X, F_{j}\right)
\end{gathered}
$$

is a Fredholm operator of index zero, [2], Theorem (5.12). Here $\gamma_{0}$ is restriction to the boundary; $p_{F_{j}}$ is projection onto the subbundle $F_{j}$. Composing $\tilde{A}$ from the left with a parametrix to $R_{2}$ and from the right with $R_{1}$ we obtain an operator with the same index as $A$ which is an endomorphism of $C^{\infty}(X, \underline{N})$.

1.2. Symbols. We consider an operator $A$ as in (1), with $E_{1}=E_{2}=E, F_{1}=F_{2}=F$. The pseudodifferential principal symbol $\sigma(A)$ of $A$ is defined to be the principal symbol of $P$, restricted to $S^{*} X$. The principal boundary symbol of $A$ is a smooth section from $S^{*} \partial X$ into the endomorphisms of

$$
\pi_{\partial}^{*}\left(\left.L^{2}\left(\mathbb{R}_{+}\right) \otimes E\right|_{\partial X}\right) \oplus \pi_{\partial}^{*} F \cong\left(\left.L^{2}\left(\mathbb{R}_{+}\right) \otimes \pi_{\partial}^{*} E\right|_{\partial X}\right) \oplus \pi_{\partial}^{*} F
$$

where $\pi_{\partial}: S^{*} \partial X \rightarrow \partial X$ is the canonical projection. It is best described for a trivial onedimensional bundle and in local coordinates $\left(x^{\prime}, x_{n}, \xi^{\prime}, \xi_{n}\right)$ for $T^{*} X$ in a neighborhood of the boundary. Here, $G$ acts like a pseudodifferential operator along the boundary, with an operator-valued symbol taking values in regularizing operators in the normal direction. One way to write this operator-valued symbol is via a so-called symbol kernel $\tilde{g}=\tilde{g}\left(x^{\prime}, \xi^{\prime}, x_{n}, y_{n}\right)$. For fixed $\left(x^{\prime}, \xi^{\prime}\right)$, this is a rapidly decreasing function in $x_{n}$ and $y_{n}$ which acts as an integral operator on $L^{2}\left(\mathbb{R}_{+}\right)$. It satisfies special estimates, combining the usual pseudodifferential estimates in $x^{\prime}$ and $\xi^{\prime}$ with those for rapidly decreasing functions in $x_{n}$ and $y_{n}$. The singular Green symbol $g$ of $G$ is defined from the symbol kernel via Fourier and inverse Fourier transform:

$$
g\left(x^{\prime}, \xi^{\prime}, \xi_{n}, \eta_{n}\right)=F_{x_{n} \rightarrow \xi_{n}} \bar{F}_{y_{n} \rightarrow \eta_{n}} \tilde{g}\left(x^{\prime}, \xi^{\prime}, x_{n}, y_{n}\right)
$$

It has an expansion into homogeneous terms; the leading one we call $g_{0}$. Inverting the operation above, we associate with $g_{0}$ a symbol-kernel $\tilde{g}_{0}\left(x^{\prime}, \xi^{\prime}, x_{n}, y_{n}\right)$ which is rapidly decreasing in $x_{n}$ and $y_{n}$ for fixed $\left(x^{\prime}, \xi^{\prime}\right)$. We denote by $g_{0}\left(x^{\prime}, \xi^{\prime}, D_{n}\right)$ the (compact) operator induced on $L^{2}\left(\mathbb{R}_{+}\right)$by this kernel. Similarly, $K$ and $T$ have symbol-kernels $\tilde{k}\left(x^{\prime}, \xi^{\prime}, x_{n}\right)$ and $\tilde{t}\left(x^{\prime}, \xi^{\prime}, y_{n}\right)$; these are rapidly decreasing functions for fixed $\left(x^{\prime}, \xi^{\prime}\right)$. The symbols $k$ and $t$ are defined as their Fourier and inverse Fourier transforms. They have asymptotic expansions with leading terms $k_{0}$ and $t_{0}$. Via the symbol-kernels 
$\tilde{k}_{0}$ and $\tilde{t}_{0}$ one defines $k_{0}\left(x^{\prime}, \xi^{\prime}, D_{n}\right): \mathbb{C} \rightarrow L^{2}\left(\mathbb{R}_{+}\right)$as multiplication by $\tilde{k}_{0}\left(x^{\prime}, \xi^{\prime}, \cdot\right)$, while $t_{0}\left(x^{\prime}, \xi^{\prime}, D_{n}\right): L^{2}\left(\mathbb{R}_{+}\right) \rightarrow \mathbb{C}$ is the operator $\varphi \mapsto \int \tilde{t}_{0}\left(x^{\prime}, \xi^{\prime}, \cdot\right) \varphi$.

We denote by $p_{0}$ and $s_{0}$ the principal symbols of $P$ and $S$, respectively. The boundary symbol $\gamma(A)$ of $A$ in $\left(x^{\prime}, \xi^{\prime}\right)$ is then defined by

$$
\gamma(A)\left(x^{\prime}, \xi^{\prime}\right)=\left(\begin{array}{cc}
p_{0}\left(x^{\prime}, 0, \xi^{\prime}, D_{n}\right)_{+}+g_{0}\left(x^{\prime}, \xi^{\prime}, D_{n}\right) & k_{0}\left(x^{\prime}, \xi^{\prime}, D_{n}\right) \\
t_{0}\left(x^{\prime}, \xi^{\prime}, D_{n}\right) & s_{0}\left(x^{\prime}, \xi^{\prime}\right)
\end{array}\right) .
$$

Two things are important to note:

(i) Except for $p_{0}\left(x^{\prime}, 0, \xi^{\prime}, D_{n}\right)$ all entries in $\gamma(A)\left(x^{\prime}, \xi^{\prime}\right)$ are compact.

(ii) The boundary symbol is 'twisted' homogeneous of degree zero in the sense that

$$
\left(\begin{array}{cc}
\kappa_{\lambda^{-1}} & 0 \\
0 & \text { id }
\end{array}\right) \gamma(A)\left(x^{\prime}, \lambda \xi^{\prime}\right)\left(\begin{array}{cc}
\kappa_{\lambda} & 0 \\
0 & \text { id }
\end{array}\right)=\gamma(A)\left(x^{\prime}, \xi^{\prime}\right), \quad \lambda>0,
$$

with the $L^{2}\left(\mathbb{R}_{+}\right)$-unitary $\kappa_{\lambda}$ given by $\kappa_{\lambda} f(t)=\sqrt{\lambda} f(\lambda t)$.

1.3. Kernel and range of the boundary symbol map. The algebra $\mathfrak{A}$ contains the ideal $\mathfrak{I}$ given by the $C^{*}$-closure of all elements of the form

$$
\left(\begin{array}{cc}
\varphi P \psi+G & K \\
T & S
\end{array}\right)
$$

with $\varphi, \psi$ in $C_{c}^{\infty}\left(X^{\circ}\right)$ and $G, K, T, S$ of negative order and class zero. Clearly, $\mathfrak{I}$ is contained in the kernel of $\gamma$. More is true:

Theorem 3. The kernel of the boundary symbol map $\gamma$ is equal to $\mathfrak{I}$. The quotient $\mathfrak{I} / \mathfrak{S}$ is isomorphic to $C_{0}\left(S^{*} X^{\circ}\right.$, End $\left.\pi^{*} E\right)$ with isomorphism induced by the principal symbol. Here $\pi^{*} E$ is the pull-back of $E$ under the projection $\pi: S^{*} X^{\circ} \rightarrow X^{\circ}$.

Proof. This is immediate from the considerations for the case of trivial bundles [7], Theorem 1.

In order to make the computation of the range of $\gamma$ more transparent, let us first consider the localized situation with $E$ and $F$ trivial one-dimensional. We write $\gamma$ as a $2 \times 2$ matrix with entries $\gamma_{i j}, i, j=1,2$.

Let $p$ be a classical pseudodifferential symbol of order zero on $\mathbb{R}^{n}$. For fixed $\left(x^{\prime}, \xi^{\prime}\right)$, $p_{0}\left(x^{\prime}, 0, \xi^{\prime}, \xi_{n}\right)$ is a symbol of order zero on $\mathbb{R}$. The transmission property assures that the values of $p$ in $\xi_{n}=+\infty$ and $\xi_{n}=-\infty$ coincide. The operator

$$
p_{0}\left(x^{\prime}, 0, \xi^{\prime}, D_{n}\right)_{+}=r^{+} \text {op } p_{0}\left(x^{\prime}, 0, \xi^{\prime}, \xi_{n}\right) e^{+}: L^{2}\left(\mathbb{R}_{+}\right) \rightarrow L^{2}\left(\mathbb{R}_{+}\right)
$$

in the upper left corner $\gamma_{11}$ then is a Toeplitz type operator. In fact, it is unitarily equivalent to the usual Toeplitz operator $T_{f}$ with symbol $f(z)=p_{0}\left(x^{\prime}, 0, \xi^{\prime}, i(z-1) /(z+1)\right)$. Thus 
the image of the upper left corner under the boundary symbol map is a subalgebra of $C\left(S^{*} \partial X, \mathfrak{I}\right)$, where $\mathfrak{I}$ denotes the Toeplitz algebra.

All other entries in the matrix for $\gamma(A)\left(x^{\prime}, \xi^{\prime}\right)$ are compact, so that the boundary symbol is, for fixed $\left(x^{\prime}, \xi^{\prime}\right)$, a so-called Wiener-Hopf operator on $L^{2}\left(\mathbb{R}_{+}\right) \oplus \mathbb{C}$. One might conjecture that the range of the boundary symbol map consisted of all sections with values in Wiener-Hopf operators. It came as a surprise (and turned out to be a crucial fact) in [7] that this is not the case. It is the range of the upper left corner $\gamma_{11}$ which is slightly smaller than expected: Let us denote by $\mathfrak{I}_{0}$ the subalgebra of those Toeplitz operators whose symbol vanishes in $z=-1$ (corresponding to $\xi_{n}= \pm \infty$ ). The range of $\gamma_{11}$ contains as an ideal all sections of $S^{*} \partial X$ with values in $\mathfrak{I}_{0}$, but the only sections of the form $g\left(x^{\prime}, \xi^{\prime}\right) \otimes \operatorname{Id}_{L^{2}\left(\mathbb{R}_{+}\right)}$it contains are those where $g$ is independent of $\xi^{\prime}$, thus a function on $\partial X$, not $S^{*} \partial X$. We therefore get a split short exact sequence of $C^{*}$-algebras

$$
0 \rightarrow C\left(S^{*} \partial X, \mathfrak{I}_{0}\right) \rightarrow \operatorname{im} \gamma_{11} \rightarrow C(\partial X) \rightarrow 0
$$

Let us now go over to the case of general bundles, considering the entries in the matrix for $\gamma(A)\left(x^{\prime}, \xi^{\prime}\right)$ separately, writing $\tilde{E}$ and $\tilde{F}$ instead of $\pi_{\partial}^{*}\left(\left.E\right|_{\partial X}\right)$ and $\pi_{\partial}^{*} F$ :

(i) The boundary symbol $t_{0}\left(\cdot, \cdot, D_{n}\right)$ is a continuous section from $S^{*} \partial X$ to $\operatorname{Hom}\left(L^{2}\left(\mathbb{R}_{+}\right) \otimes \tilde{E}, \tilde{F}\right)$ given by integration against the symbol kernel of $t_{0}$, hence a section of $\mathscr{B}\left(L^{2}\left(\mathbb{R}_{+}\right), \mathbb{C}\right) \otimes \operatorname{Hom}(\tilde{E}, \tilde{F})$. The construction in [7], Lemma 4 shows that all elements in that space are obtained that way.

(ii) Similarly, the range of the closure of the boundary symbol map for the Poisson operators of order zero consists of all continuous sections from $S^{*} \partial X$ to $\mathscr{B}\left(\mathbb{C}, L^{2}\left(\mathbb{R}_{+}\right)\right) \otimes \operatorname{Hom}(\tilde{F}, \tilde{E})$.

(iii) The boundary symbols of zero order pseudodifferential operators along the boundary are their principal symbols, thus certain elements of $C(\partial X$, End $\tilde{F})$, and in fact, all elements in this space are obtained as such symbols.

(iv) The continuous sections from $S^{*} \partial X$ into $\pi_{\partial}^{*}\left(\mathfrak{I}_{0} \otimes \operatorname{End}\left(\left.E\right|_{\partial X}\right)\right) \cong \mathfrak{I}_{0} \otimes \operatorname{End} \tilde{E}$ are contained as an ideal in the range of $\gamma_{11}$ (the upper left corner of $\gamma$ ) by a bundle valued analog of [7], Lemma 5, while, as in [7], Lemma 6,

$$
\operatorname{im} \gamma_{11} \cap C\left(S^{*} \partial X, \text { End } \tilde{E}\right)=C\left(\partial X, \text { End }\left.E\right|_{\partial X}\right)
$$

Here, we consider the elements of $C\left(\partial X\right.$, End $\left.\left.E\right|_{\partial X}\right)$ and $C\left(S^{*} \partial X\right.$, End $\left.\tilde{E}\right)$ as elements of $C\left(S^{*} \partial X, \operatorname{End}\left(L^{2}\left(\mathbb{R}_{+}\right) \otimes \tilde{E}\right)\right)$ by acting as the identity on $L^{2}\left(\mathbb{R}_{+}\right)$. We conclude that we get a split short exact sequence of $C^{*}$-algebras

$$
0 \rightarrow C\left(S^{*} \partial X, \mathfrak{I}_{0} \otimes \text { End } \tilde{E}\right) \rightarrow \operatorname{im} \gamma_{11} \rightarrow C\left(\partial X, \text { End }\left.E\right|_{\partial X}\right) \rightarrow 0
$$

We can now define the subbundle $\tilde{\mathfrak{W}}_{0}$ of endomorphisms of (7), consisting of all $2 \times 2$ matrices $w=\left(w_{i j}\right)_{i, j=1,2}$ with $w_{11} \in \mathfrak{I}_{0} \otimes \operatorname{End} \tilde{E}, w_{12} \in \mathscr{B}\left(\mathbb{C}, L^{2}\left(\mathbb{R}_{+}\right)\right) \otimes \operatorname{Hom}(\tilde{F}, \tilde{E})$, $w_{21} \in \mathscr{B}\left(L^{2}\left(\mathbb{R}_{+}\right), \mathbb{C}\right) \otimes \operatorname{Hom}(\tilde{E}, \tilde{F})$, and $w_{22} \in \operatorname{End} \tilde{F}$. We obtain: 
Theorem 4. The image of $\gamma$ fits into the following split exact sequence of $C^{*}$-algebras:

$$
0 \rightarrow C\left(S^{*} \partial X, \tilde{\mathfrak{B}}_{0}\right) \rightarrow \operatorname{im} \gamma \rightarrow C\left(\partial X, \operatorname{End}\left(\left.E\right|_{\partial X}\right)\right) \rightarrow 0 .
$$

We note that $\tilde{\mathfrak{W}}_{0}$ is the bundle valued analog of the algebra $\mathfrak{W}_{0}$ in [7]; for $E=F=\mathbb{C}$ both coincide. Strong Morita equivalence (as discussed in [7], Section 1.5) together with the fact that $\mathfrak{I}_{0}$ has vanishing $K$-theory therefore implies (cf. [7], Lemma 7 ):

Lemma 5. $K_{i}\left(C\left(S^{*} \partial X, \tilde{\mathfrak{W}}_{0}\right)\right)=0, i=0,1$.

The split in $(8)$ is implemented by the $C^{*}$-algebra homomorphism

$$
b: C\left(\partial X, \operatorname{End}\left(\left.E\right|_{\partial X}\right)\right) \rightarrow \operatorname{im} \gamma, \quad g \mapsto \gamma\left(\left(\begin{array}{ll}
f & 0 \\
0 & 0
\end{array}\right)\right),
$$

where $f$ is any continuous section in $C(X$, End $E)$ with $\left.f\right|_{\partial X}=g$. We then conclude as in [7], Corollary 8:

Corollary 6. The induced homomorphism is an isomorphism

$$
b_{*}: K_{i}\left(C\left(\partial X, \operatorname{End}\left(\left.E\right|_{\partial X}\right)\right)\right) \rightarrow K_{i}(\operatorname{im} \gamma)=K_{i}(\mathfrak{A} / \mathfrak{J}) .
$$

\section{2. $K$-theory preliminaries}

Definition 7. Let $A$ be a $C^{*}$-algebra. The cone over $A$ is the $C^{*}$-algebra $C A:=\{f:[0,1] \rightarrow A \mid f(1)=0\}$.

Since $C A$ is a contractible $C^{*}$-algebra, its $K$-theory vanishes. The suspension of $A$ is given by $S A:=\{f \in C A \mid f(0)=0\}$.

Definition 8. If $f: B \rightarrow A$ is a $C^{*}$-algebra homomorphism, the mapping cone $C f$ is defined to be $C f:=\{(b, \phi) \in B \oplus C A ; f(b)=\phi(0)\}$.

Projection onto $B$ defines a short exact sequence

$$
0 \rightarrow S A \stackrel{\hat{\imath}}{\rightarrow} C f \stackrel{q}{\rightarrow} B \rightarrow 0
$$

The assignment of this exact sequence to each $C^{*}$-algebra homomorphism $f$ defines a functor between the corresponding categories (whose morphisms consist of commutative diagrams of homomorphisms or of exact sequences, respectively). This functor is exact; i.e. we have:

Lemma 9. Assume that the following is a commutative diagram of short exact sequences of $C^{*}$-algebras:

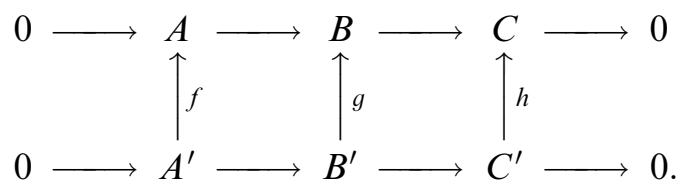


Then we get an induced commutative grid of short exact sequences of $C^{*}$-algebras:

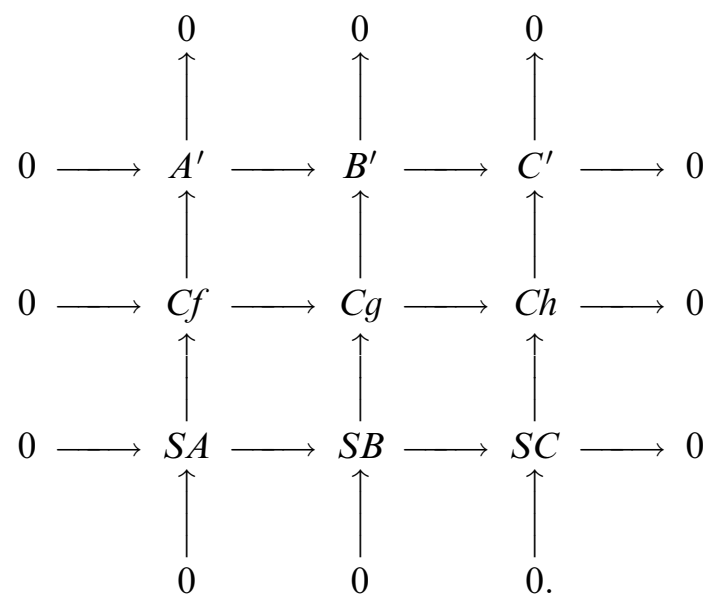

Lemma 9 can be proven by a diagram chase, using that the maps $C B \rightarrow C C$ and $S B \rightarrow S C$ are surjective (the exactness of $S$ is proven in [9], Proposition 10.1.2).

Lemma 10. The exact sequence (9) induces six-term cyclic exact sequences in $K$-theory, whose connecting mappings $K_{i}(B) \rightarrow K_{1-i}(S A)$ become, under the canonical isomorphisms $K_{1-i}(S A) \stackrel{\cong}{\rightrightarrows} K_{i}(A)$, the mappings induced by $f$.

More precisely, this lemma states that the two diagrams

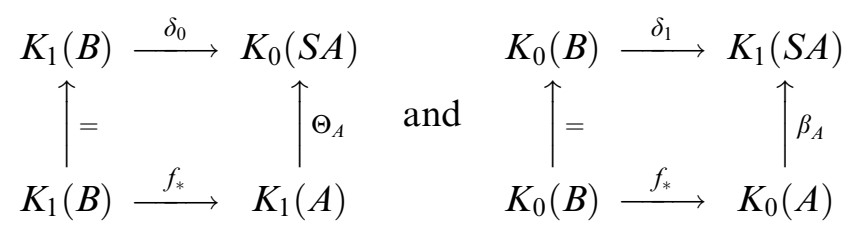

commute, where $\delta_{0}$ and $\delta_{1}$ denote, respectively, the index and the exponential mappings [9], 9.1.3 and 12.1.1, induced by (9), $\Theta_{A}$ is the isomorphism defined in [9], 10.1.3, and $\beta_{A}$ is the Bott isomorphism [9], 11.1.1. This follows by applying naturality of the long exact sequences in $K$-theory to the diagram

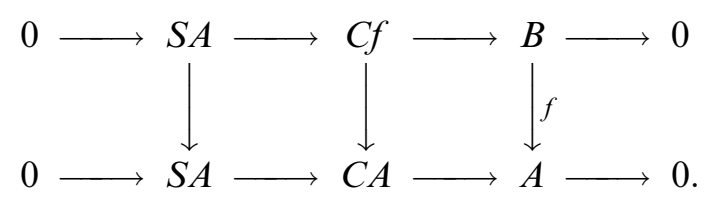

Lemma 11. If $f: B \rightarrow A$ is a surjective $C^{*}$-homomorphism, then the map $j: \operatorname{ker} f \ni x \mapsto(x, 0) \in C f$ induces a $K$-theory isomorphism, which fits into the commutative diagram

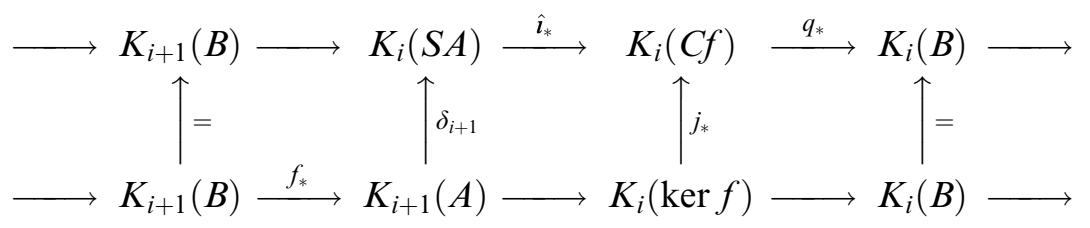


where the upper row is the cyclic exact sequence induced by (9), and the lower one is that induced by

$$
0 \rightarrow \operatorname{ker} f \rightarrow B \stackrel{f}{\rightarrow} A \rightarrow 0
$$

Proof. This result is certainly well known. For the sake of completeness, and since we did not find a convenient reference, we sketch the arguments. One proves that $j_{*}$ is a $K$ theory isomorphism using that $K_{*}(C A)=0$ in the cyclic exact sequence associated to the short exact sequence

$$
0 \rightarrow \operatorname{ker} f \stackrel{j}{\rightarrow} C f \rightarrow C A \rightarrow 0
$$

induced by projection of $C f$ onto the second coordinate. The boundary map $\delta_{i+1}$ is well known to be an isomorphism. It remains to establish commutativity. The commutativity of the left rectangle is part of Lemma 10. The right rectangle commutes by naturality of the $K$-theory functor, since $q \circ j=(i: \operatorname{ker} f \hookrightarrow B)$. The argument for the middle square is a little more involved. Observe that $j_{*}^{-1} \hat{\boldsymbol{l}}_{*} \delta_{i+1}$ defines another homomorphism $K_{i+1}(A) \rightarrow K_{i}(\operatorname{ker} f)$ which is, by the naturality of all constructions, natural and makes the $K$-theory sequence of the short exact sequence of $C^{*}$-algebras exact.

However, homomorphisms with this properties are defined uniquely (up to a universal sign) [12], Exercise 9.F, therefore the diagram is commutative up to this sign. The special exact sequence $0 \rightarrow S A \rightarrow C A \rightarrow A \rightarrow 0$ shows that this sign is +1 .

\section{3. $K$-theory of Boutet de Monvel's algebra}

In order to keep the notation simple, we shall write $C_{0}\left(X^{\circ}\right), C(X)$, and $C(\partial X)$ instead of $C_{0}\left(X^{\circ}, \operatorname{End}\left(\left.E\right|_{X^{\circ}}\right)\right), C(X$, End $E)$, and $C\left(\partial X, \operatorname{End}\left(\left.E\right|_{\partial X}\right)\right)$. Identifying a continuous function $f$ on $X^{\circ}$ or $X$ with the operator $\left(\begin{array}{ll}f & 0 \\ 0 & 0\end{array}\right) \in \mathfrak{A}$, where $f$ acts by multiplica-
tion, we have natural maps

$$
m_{0}: C_{0}\left(X^{\circ}\right) \rightarrow \mathfrak{I} \quad \text { and } \quad m: C(X) \rightarrow \mathfrak{A}
$$

and thus a commutative diagram of exact sequences:

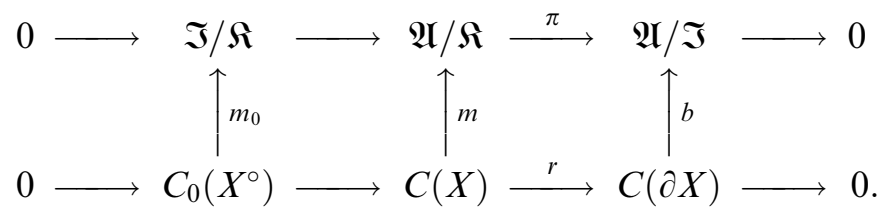

(We do not distinguish between the isomorphic $C^{*}$-algebras $\mathfrak{U} / \mathfrak{I}$ and the image of $\gamma$.) From Lemma 9 we obtain the commutative grid 
(10)

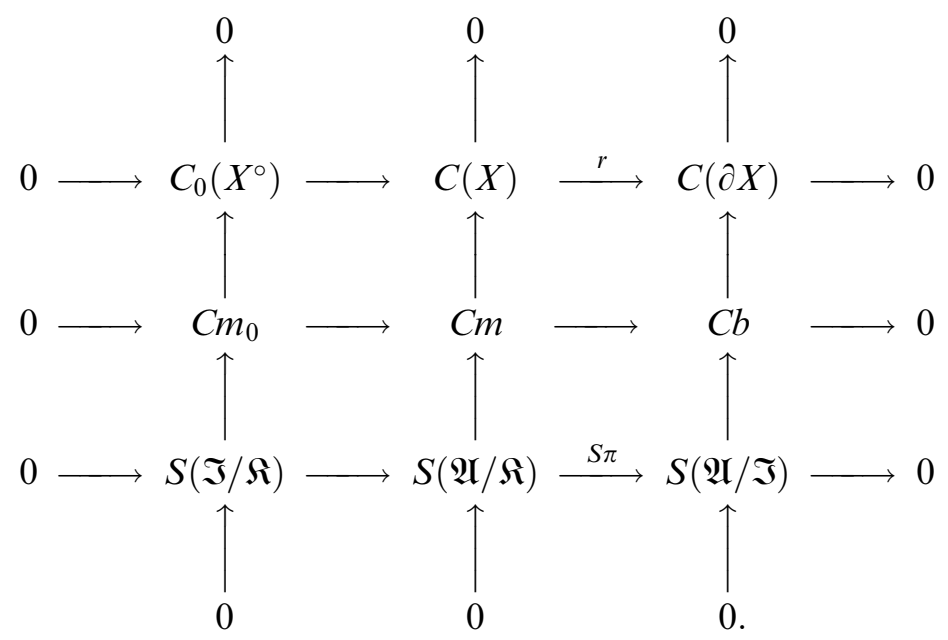

These six short exact sequences induce six cyclic long exact sequences in $K$-theory which we want to analyze next. By Corollary $6, b$ induces an isomorphism in $K$-theory. From Lemma 10 and the cyclic exact sequence of $0 \rightarrow S(\mathfrak{U} / \mathfrak{I}) \rightarrow C b \rightarrow C(\partial X) \rightarrow 0$ we therefore conclude that $K_{*}(C b)=0$. From this in turn we deduce, using the cyclic exact sequence of $0 \rightarrow \mathrm{Cm}_{0} \rightarrow \mathrm{Cm} \rightarrow \mathrm{Cb} \rightarrow 0$, that $\mathrm{Cm}_{0} \rightarrow \mathrm{Cm}$ induces an isomorphism in $K$-theory.

We therefore get the following commutative diagram of cyclic exact sequences of $K$-theory groups, again using Lemma 10 and the natural isomorphisms $K_{1-i}(S A) \cong K_{i}(A)$ :

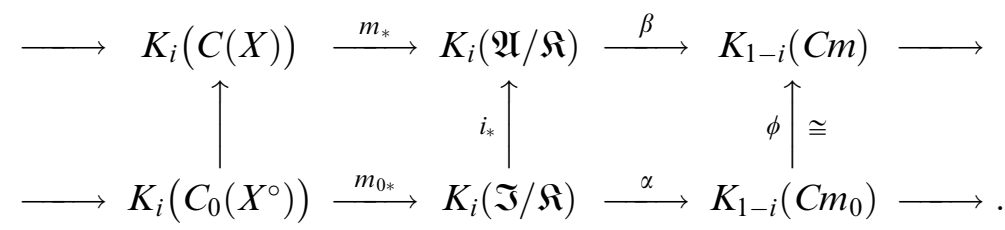

According to Theorem 3, the principal symbol provides an isomorphism $\mathfrak{I} / \mathfrak{\Re} \cong C_{0}\left(S^{*} X^{\circ}\right)$, and $m_{0}$ becomes the pull back homomorphism $\pi^{*}$ under this isomorphism. Since $X$ is connected and $\partial X \neq \emptyset$, there is a nonvanishing section of the cotangent bundle (see e.g. [7], Proposition 9, for a proof of this well-known fact), which yields a map $C_{0}\left(S^{*} X^{\circ}\right) \rightarrow C\left(X^{\circ}\right)$. Therefore $m_{0 *}$ has a split $s$ (which is not necessarily natural). Consequently, $\alpha$ also has a split $s^{\prime}$. Define now $s^{\prime \prime}:=i_{*} \circ s^{\prime} \circ \phi^{-1}: K_{1-i}(C m) \rightarrow K_{i}(\mathfrak{A} / \mathfrak{A})$. An easy diagram chase shows that $s^{\prime \prime}$ is a split of $\beta$. Consequently, our long exact sequence yields natural short exact sequences

$$
0 \longrightarrow K_{i}(C(X)) \stackrel{m_{*}}{\longrightarrow} K_{i}(\mathfrak{U} / \mathfrak{R}) \stackrel{\beta}{\longrightarrow} K_{1-i}(C m) \longrightarrow 0
$$

which have a (not necessarily natural) split. In particular, each element in $K_{i}(\mathfrak{A} / \mathfrak{R})$ can be written as the sum of two elements, one in the range of $m_{*}$ and one in the range of $s^{\prime \prime}$, thus in the range of $i_{*}$.

It remains to identify $K_{1-i}(\mathrm{Cm}) \cong K_{1-i}\left(\mathrm{Cm}_{0}\right)$. For this, recall the natural short exact sequence for the ball- or disc — completion of the cotangent bundle, extended to a commutative diagram 


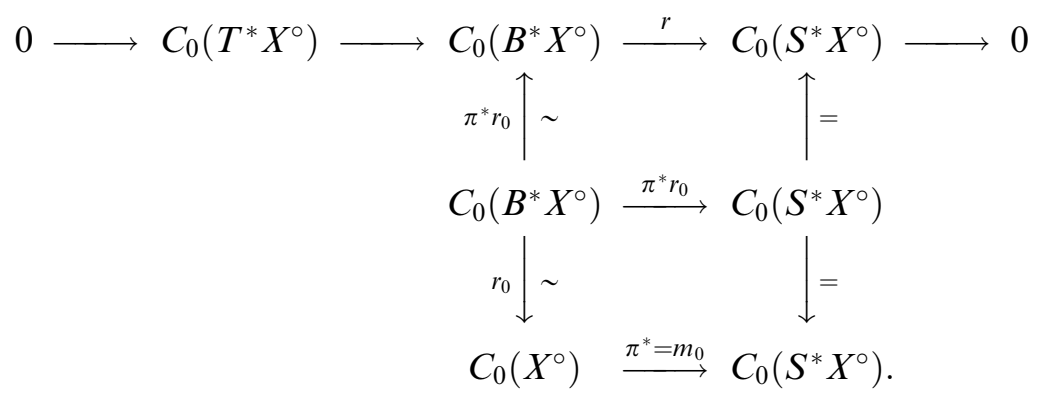

Here, $\pi^{*}$ denotes pull back from the base to the total space of the bundle, and $r$ and $r_{0}$ denote restriction to the boundary of the disc bundle, or the zero section of the disc bundle, respectively; $\sim$ denotes homotopy equivalences of $C^{*}$-algebras. Again we have omitted the bundles from the notation.

We get induced short exact mapping cone sequences

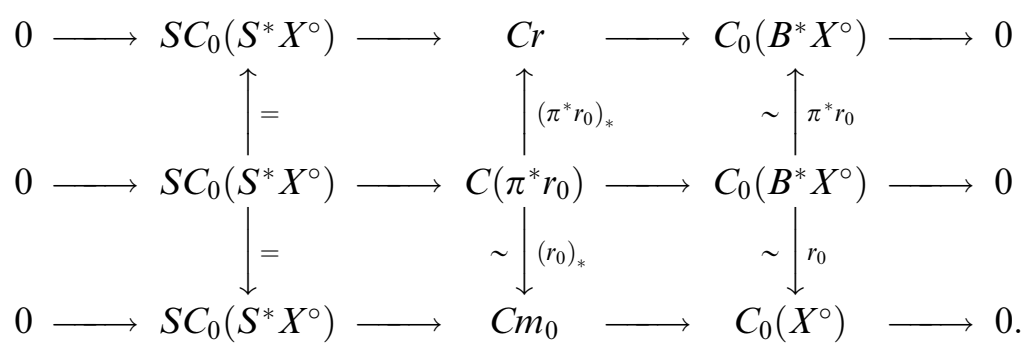

The corresponding cyclic exact $K$-theory sequences together with the 5-lemma imply that the induced maps between the mapping cones induce isomorphisms in $K$-theory. diagram

Finally, since $r$ is surjective and $\operatorname{ker} r=C_{0}\left(T^{*} X^{\circ}\right)$, Lemma 11 yields the commutative

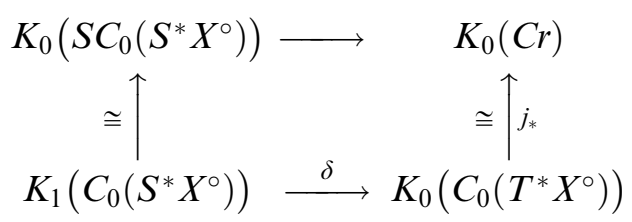

where the lower horizontal arrow is the index mapping for the first row in (12), and the upper horizontal is induced by the first row in (13).

The composition of all these maps gives a natural way to identify $K_{i}(\mathrm{Cm})$ with $K_{i}\left(C_{0}\left(T^{*} X^{\circ}\right)\right)$. This already finishes the proof of Theorem 1. A more detailed explanation of this step will be needed below, in the proof of Theorem 2 .

\section{Index theory}

We consider the commutative diagram 


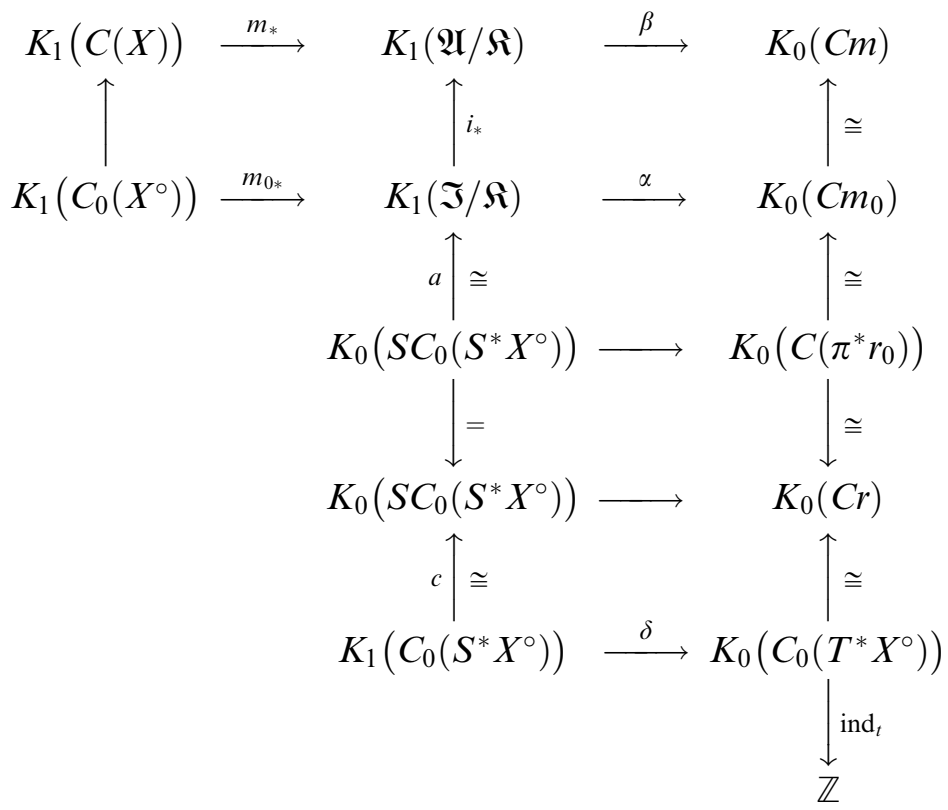

where the first two rows are portions of (11). The second, third and fourth rows in (15) are portions of the cyclic sequences associated to (13) (notice that, if we use the isomorphism $\mathfrak{I} / \mathfrak{R} \cong C_{0}\left(S^{*} X^{\circ}\right)$ as an identification, then the first column in (10) is equal to the last row in (13)), while the fourth and fifth rows are just (14). Note that the composed isomorphism $c^{-1} a^{-1}: K_{1}(\mathfrak{I} / \mathfrak{R}) \rightarrow K_{1}\left(C_{0}\left(S^{*} X^{\circ}\right)\right)$ in the left row is exactly the map induced by the interior symbol.

Definition 12. The map $p$ in (5) is the composition of all the maps (reverting arrows

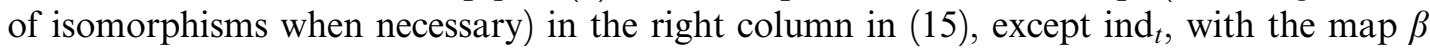
from $K_{1}(\mathfrak{U} / \mathfrak{R})$ to $K_{0}(\mathrm{Cm})$ in the first row.

Remark 13. The definition of $p$ uses the inverse of the isomorphism $K_{0}\left(C m_{0}\right) \rightarrow K_{0}(\mathrm{Cm})$, which we can not write down explicitly-our argument which proves that the map is an isomorphism is actually rather indirect.

Equivalently, the problem can be restated as replacing a given invertible element of $\mathfrak{A} / \mathfrak{R}$ by the sum of elements in the images of $m_{*}$ and $i_{*}$, respectively, representing the same element in $K_{1}$. That this is possible is based on the same indirect argument which shows that $K_{0}\left(\mathrm{Cm}_{0}\right) \rightarrow K_{0}(\mathrm{Cm})$ is an isomorphism, cf. the argument right after (11). Nevertheless, we will see below, in our proof of Fedosov's index formula (3), that this representation is actually very useful.

To prove Theorem 2, namely that $\operatorname{ind}_{t} \circ p$ and the Fredholm index are equal on $K_{1}(\mathfrak{A} / \mathfrak{K})$, it is enough to show that they are equal on the image of $m_{*}$ and on the image of $i_{*}$. On the image of $m_{*}$, both are zero: On one hand, the range of $m_{*}$ consists of equivalence classes (modulo $\mathfrak{R}$ ) of invertible multiplication operators. Each of these has index zero. On the other hand, the first row in (15) is exact, thus the range of $m_{*}$ is mapped to zero. The commutativity of (15) then shows that all we have to prove is that ind $_{t} \circ \delta \circ c^{-1} \circ a^{-1}$ is the Fredholm index. 
For that let $\Psi$ denote the $C^{*}$-closure of the algebra of all classical pseudodifferential operators of order 0 on $\tilde{X}$ in the algebra of all bounded operators on $L^{2}(\tilde{X})$. The zero extension on the orthogonal complement of $L^{2}(X)$ in $L^{2}(\tilde{X})$ defines a $*$-homomorphism $\mathfrak{I}_{11} \rightarrow \Psi$, where $\mathfrak{I}_{11}$ denotes the ideal formed by the upper-left corners of $\mathfrak{I}$. That gives us a commutative diagram of exact sequences:

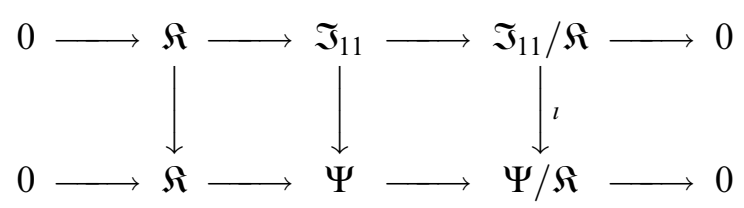

where we have denoted by the same symbol the compact ideal in the bounded operators on $L^{2}(X)$ and on $L^{2}(\tilde{X})$. The canonical injection of $\mathfrak{I}_{11}$ into $\mathfrak{I}$ induces an isomorphism between $\mathfrak{I}_{11} / \mathfrak{S}$ and $\mathfrak{I} / \mathfrak{S}$ (see comments right before Theorem 1 in [7]). That isomorphism and the naturality of the index mapping for (16) then imply that

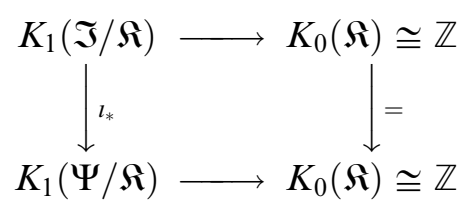

commutes, where the horizontal arrows are the Fredholm-index homomorphisms for $\mathfrak{I}$ and for $\Psi$.

For any closed manifold, the principal symbol induces an isomorphism between $\Psi / \mathfrak{R}$ and the continuous functions on the cosphere bundle. This follows from the classical estimate for the norm, modulo compacts, of a pseudo-differential operator [6], Theorem A.4. We therefore have $K_{1}(\Psi / \mathfrak{R}) \cong K_{1}\left(C\left(S^{*} \tilde{X}\right)\right)$. Modulo this isomorphism, the Atiyah-Singer index theorem [1] states that the Fredholm-index homomorphism for $\Psi$ is the composition of the topological index $\operatorname{ind}_{t}^{\tilde{X}}: K_{0}\left(C_{0}\left(T^{*} \tilde{X}\right)\right) \rightarrow \mathbb{Z}$ with the index mapping for the exact sequence

$$
0 \rightarrow C_{0}\left(T^{*} \tilde{X}\right) \rightarrow C\left(B^{*} \tilde{X}\right) \rightarrow C\left(S^{*} \tilde{X}\right) \rightarrow 0
$$

(see e.g. [7], Proposition 15, for a proof that the classical difference bundle construction indeed gives the $C^{*}$-algebra $K$-theory index mapping for this sequence).

Now consider the commutative diagram of exact sequences

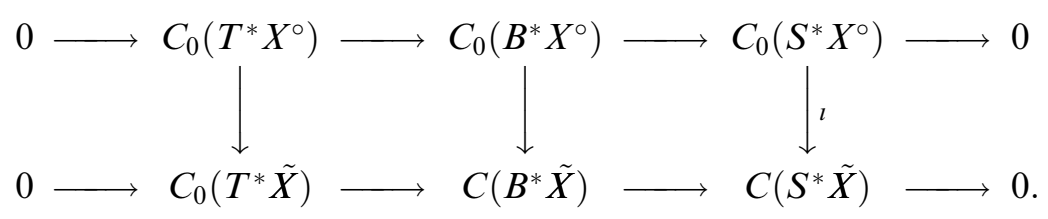

By naturality of the index map, the following diagram commutes: 


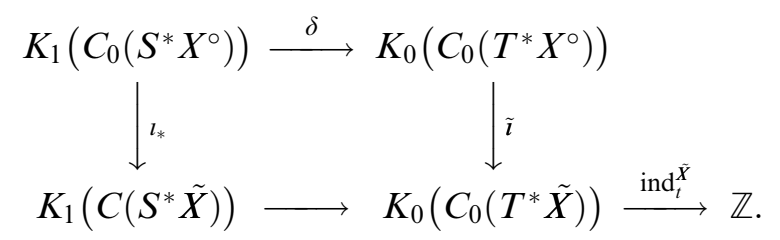

The Atiyah-Singer index theorem and the commutativity of (17) imply that the composition of the two lower horizontal and the left vertical arrow in (19) gives the Fredholm in$\operatorname{dex}$, hence that $\operatorname{ind}_{t}^{\tilde{X}} \circ \tilde{\imath} \circ \delta$ is the Fredholm index for $\mathfrak{J}$. This proves (6) since, by definition, $\operatorname{ind}_{t}=\operatorname{ind}_{t}^{\tilde{X}} \circ \tilde{\imath}$.

To show the last statement, $p=$ ind, look again at (15). As before, it is enough to prove that ind $\circ i_{*}=p \circ i_{*}$ and ind $\circ m_{*}=p \circ m_{*}$. Our diagram gives $p \circ i_{*}=\delta$ and $p \circ m_{*}=0$, while ind $\circ i_{*}=\delta$ and ind $\circ m_{*}=0$ are proven in [7], Lemmas 16 and 17. This shows Theorem 2.

It remains to check the validity of Fedosov's index formula (3). His proof of homotopy invariance in [3], Proof of Theorem 2.4 in Chapter II, shows that the expression only depends on the $K_{1}$-class represented by the elliptic operator $A$ in $K_{1}(\mathfrak{A} / \mathfrak{R})$, because the formula is clearly additive for the block sum addition. This can be considered to be the heart of the proof of the formula, and we do not offer a new proof for it. Once we know that Fedosov's formula defines a homomorphism from $K_{1}(\mathfrak{A} / \mathfrak{R})$, we can identify it easily with the index map: It is clear that the formula is zero for multiplication operators, i.e. elements in the image of $K_{1}(C(X))$. Because of Theorem 2 (compare also the proof above), it suffices to check Formula (3) for operators $A$ supported in the interior. But for those, the formula reduces to the classical Atiyah-Singer index formula. One can use the double of $X$ to get exactly the situation of Atiyah-Singer.

A detail hidden by the simplified notation - but needed for the naturality of the index map - is the existence of a canonical isomorphism

$$
K_{0}\left(C_{0}\left(T^{*} X^{\circ}, \operatorname{End}\left(\pi^{*} E\right)\right)\right) \rightarrow K_{0}\left(C_{0}\left(T^{*} X^{\circ}\right)\right)
$$

if $E$ is not the zero bundle. This is well-known and comes, after a series of standard arguments, from the fact that $E$ is a direct summand of a trivial bundle and that the trivial line bundle is a direct summand in a power of $E$.

Acknowledgments. Severino Melo was supported by the Brazilian agency CNPq (Processos 452780/2003-9 and 306214/2003-2). Elmar Schrohe had support from the European Research and Training Network "Geometric Analysis" (Contract HPRN-CT-19990018). He thanks Johannes Aastrup and Ryszard Nest for several helpful discussions.

\section{References}

[1] M. F. Atiyah and I. M. Singer, The index of elliptic operators. I, Ann. Math. (2) 87 (1968), 484-530.

[2] Louis Boutet de Monvel, Boundary problems for pseudo-differential operators, Acta Math. (1-2) 126 (1971), $11-51$.

[3] B. V. Fedosov, Index theorems, in: Partial differential equations, VIII, Encyclop. Math. Sci. 65, Springer, Berlin (1996), 155-251. 
[4] G. Grubb, Functional Calculus of Pseudodifferential Boundary Problems, Second Edition, Birkhäuser, Boston 1996.

[5] G. Grubb and G. Geymonat, The essential spectrum of elliptic systems of mixed order, Math. Ann. 227 (1977), 247-276.

[6] J. J. Kohn and L. Nirenberg, An algebra of pseudo-differential operators, Comm. Pure Appl. Math. 18 (1965), 269-305.

[7] S. T. Melo, R. Nest, and E. Schrohe, C $\mathrm{C}^{*}$-structure and K-theory of Boutet de Monvel's algebra, J. reine angew. Math. 561 (2003), 145-175.

[8] S. Rempel and B.-W. Schulze, Index theory of elliptic boundary problems, Akademie-Verlag, Berlin 1982.

[9] M. Rørdam, F. Larsen, and N. J. Laustsen, An Introduction to K-Theory for C*-Algebras, London Math. Soc. Stud. Texts 49, Cambridge University Press, Cambridge 2000.

[10] E. Schrohe, Fréchet algebra techniques for boundary value problems on noncompact manifolds: Fredholm criteria and functional calculus via spectral invariance, Math. Nachr. 199 (1999), 145-185.

[11] E. Schrohe, A short introduction to Boutet de Monvel's calculus, Approaches to Singular Analysis (Berlin 1999), Oper. Th. Adv. Appl. 125, Birkhäuser, Basel (2001), 85-116.

[12] N. E. Wegge-Olsen, K-theory and $\mathrm{C}^{*}$-algebras, Oxford Science Publications, The Clarendon Press, Oxford University Press, New York 1993.

Instituto de Matemática e Estatística, Universidade de São Paulo, Caixa Postal 66281, 05315-970 São Paulo e-mail: melo@ime.usp.br

Mathematisches Institut, Universität Göttingen, Bunsenstr. 3-5, 37073 Göttingen, Germany e-mail: schick@uni-math.gwdg.de

Institut für Analysis, Universität Hannover, Welfengarten 1, 30167 Hannover, Germany

e-mail: schrohe@math.uni-hannover.de

Eingegangen 5. April 2005, in revidierter Fassung 11. Oktober 2005 